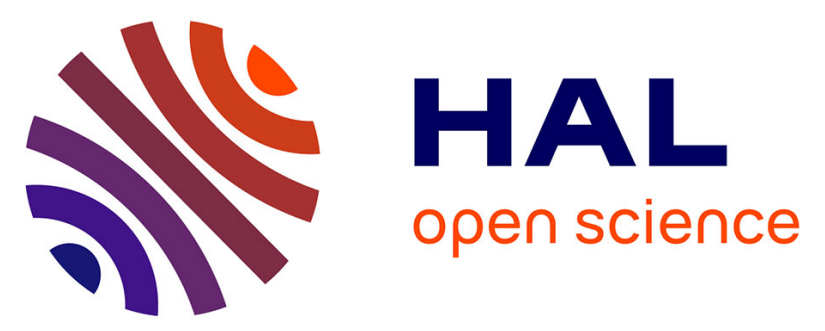

\title{
Development of new high-throughput screening method to compare and to detect efficient catalysts for adhesive materials
}

Boris Colin, Olivier Lavastre, Stéphane Fouquay, Guillaume Michaud, Frédéric Simon, Olivier Laferte, Jean-Michel Brusson

\section{To cite this version:}

Boris Colin, Olivier Lavastre, Stéphane Fouquay, Guillaume Michaud, Frédéric Simon, et al.. Development of new high-throughput screening method to compare and to detect efficient catalysts for adhesive materials. International Journal of Adhesion and Adhesives, 2016, 68, pp.47-53. 10.1016/j.ijadhadh.2016.02.002 . hal-01300878

\section{HAL Id: hal-01300878}

https://hal-univ-rennes1.archives-ouvertes.fr/hal-01300878

Submitted on 27 May 2016

HAL is a multi-disciplinary open access archive for the deposit and dissemination of scientific research documents, whether they are published or not. The documents may come from teaching and research institutions in France or abroad, or from public or private research centers.
L'archive ouverte pluridisciplinaire HAL, est destinée au dépôt et à la diffusion de documents scientifiques de niveau recherche, publiés ou non, émanant des établissements d'enseignement et de recherche français ou étrangers, des laboratoires publics ou privés. 


\title{
ACCEPTED MANUSCRIPT
}

\section{Development of new high-throughput screening method to compare and to detect efficient catalysts for adhesive materials}

\author{
Boris Colin $^{1}$, Olivier Lavastre ${ }^{1 *}$, Stéphane Fouquay ${ }^{2}$, Guillaume Michaud $^{2}$, Frédéric Simon ${ }^{2}$, \\ Olivier Laferte $^{2}$ and Jean-Michel Brusson ${ }^{3}$ \\ ${ }^{1}$ Laboratory of functional materials, IETR, UMR 6164 CNRS, University of RENNES 1, 263 Avenue du \\ Général Leclerc, 35042 Rennes Cedex, France \\ ${ }^{2}$ BOSTIK SMART TECHNOLOGY CENTER ZAC du bois de Plaisance 101, rue du Champ Cailloux, 60280 \\ Venette, France \\ ${ }^{3}$ TOTAL S.A., Tour Michelet A, 24, Cours Michelet, 92069 La Défense Cedex, France \\ *Corresponding author. Tel.: +33 223235630 ; E-mail address: olivier.lavastre@univ-rennes1.fr
}

\begin{abstract}
Organotin compounds particularly dibutyltin dilaurate, are commonly used as catalysts in coatings or adhesive materials to crosslink silyl modified polymers. However, environmental concerns should lead before 2020 to ban organotin compounds due to their high toxicity. Thus new catalysts must be developed. According to the different types of catalytic systems, i.e. acid, basic or metal containing catalysts, a large variety of candidates should be tested. Thus a high-throughput screening (HTS) method could be an interesting tool for the detection of new efficient catalysts to substitute organotin compounds. We report a global HTS method, compatible with organic amino or acid catalysts libraries, as well as with metal-containing libraries.
\end{abstract}

KEYWORDS Silyl modified polymers, Cross-linking, High-throughput screening, catalysts.

\section{INTRODUCTION}

Silyl modified polymers (SMP) are key materials, applied in many applications such as binders for elastic adhesives [1,2], composites [3,4], electrolytes [5] or for coating [6,7] applications. These hybrid polymers are also becoming increasingly common in aeronautical and aerospacial applications [8]. They can be used in several other applications due to their adjustable properties [9] such as Young's modulus, tensile strength, thermal, gas barrier and photonic properties. Cross-linking of silyl modified polymers takes place with alkoxysilane groups attached to the ends of polymer chains by hydrolysis and polycondensation with moisture [10,11]. The hydrolysis and condensation reactions occur almost simultaneously and they are in competition with each other. Organotin compounds such as dibutyltin dilaurate (DBTDL) are widely used as catalysts to accelerate the cross-linking of silyl modified polymers with moisture [12]. However, in recent years, the high toxicity of organotin compounds has been proved and the environmental concerns should lead before 2020 to ban organotin catalysts. Thus, several studies were performed in basic or acidic conditions allowing cross-linking of silyl modified polymers without tin catalysts. Indeed, strong acids like $\mathrm{CF}_{3} \mathrm{SO}_{3} \mathrm{H}$ [13] or $\mathrm{HCl}$ [14] efficiently accelerate the process of hydrolysis. Under basic conditions to deprotonate water, hydroxyl $\mathrm{OH}^{-}$ions attack the most positively charged atom by nucleophilic reactions [15]. Thus super-bases, like DBU or TBD amines are very efficient to accelerate the reaction and to increase the density of cross-linking. Metal alkoxide catalysts were also reported, in order to generate different cross-linked matrix by comparison with acid or basic organic catalysts.

In all of these studies, the discovery of efficient catalysts has always been obtained by an iterative approach. Each potential catalyst is tested one per one and sometimes at different time scale. This can be time-consuming and does not allow comparison of a large number of catalysts in the same time period, for instance for the same batch of starting material. This classical iterative method has already been described by Bostik [16] for different applications. On the other hand, the high-throughput 


\section{ACCEPTED MANUSCRIPT}

experiment is a powerful approach for the fast screening of a large diversity of compounds such as biomaterials [17], inorganic materials [18], polymers [19] or catalysts [20, 21, 22].

Here, we report a semi-quantitative, fast and inexpensive high-throughput screening method based on the visual difference of polymer surfaces when undergoing cross-linking reactions. Indeed, several steps appear during cross-linking reaction (Fig. 1). Prepolymers were used here as starting materials. These stable materials are reactive intermediates which can be cross-linked later. At the beginning of the hydrolysis process, the prepolymer is viscous and when condensation occurs, the viscosity increases. Then, a three dimensional cross-linked structure is obtained. At the end of polycondensation, the surface is solid with a gum texture. According to this point of view, the efficiency of a catalyst could be determined just by touching the surface after a given time t. Indeed, a catalyst will be efficient when the surface is not tacky with a solid gum texture at time t. On the other hand, if the mixture is viscous at time t, the catalyst is inefficient or not sufficiently efficient for the target time t.
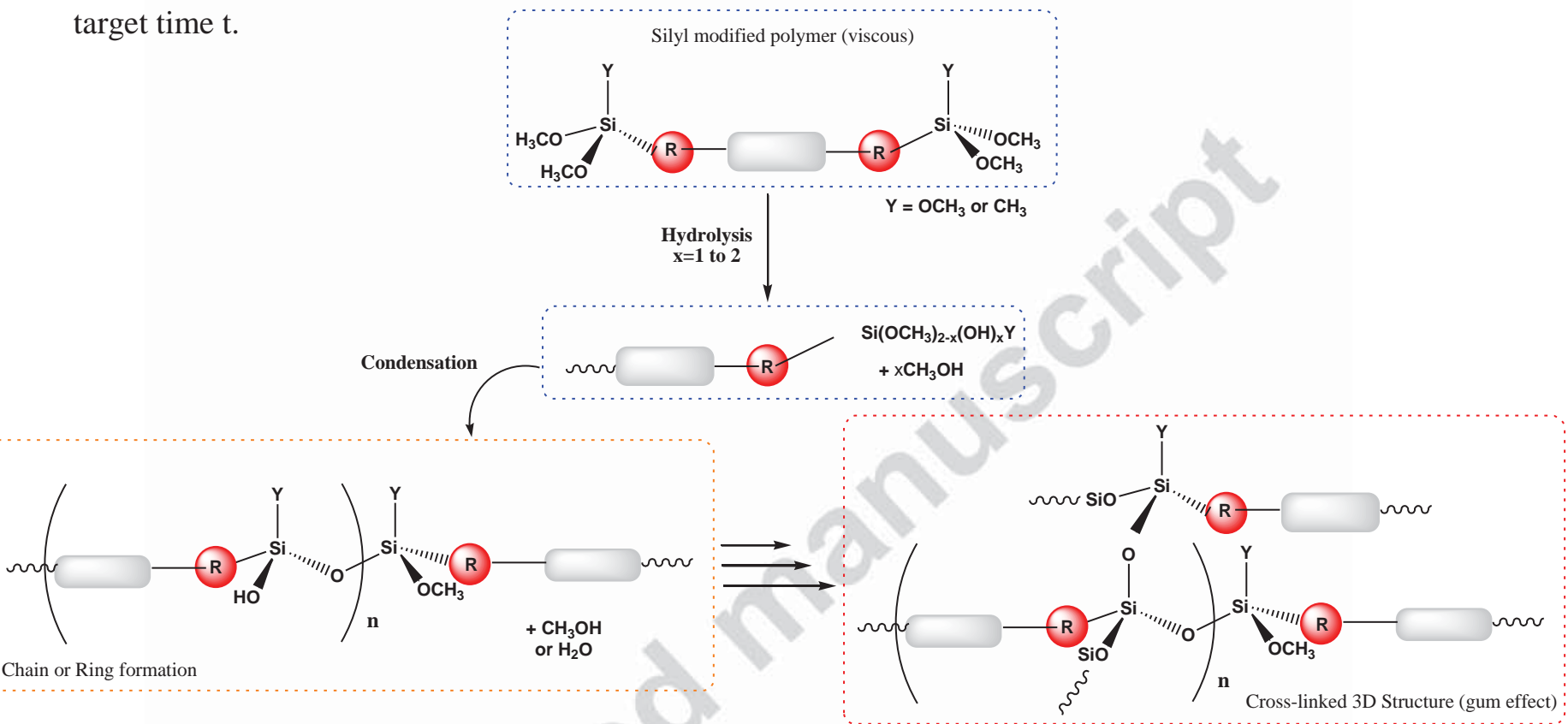

Fig. 1: General scheme of hydrolysis and condensation reactions and intermediate states: viscous or solid 3D cross-linked gum.

\section{EXPERIMENTAL}

\subsection{Adhesive materials}

Silyl modified polymers are silane based chemicals that contain inorganic and organic moieties which can form polymers exhibiting the beneficial characteristics of both often without many of their disadvantages. Two prepolymers were used for the study: SMP1 and SMP2 which were provided by BOSTIK. They are dimethoxymethylsilane terminated polyethers with a urethane moiety for SMP1 and an alkyl chain for SMP2 (Fig. 2).

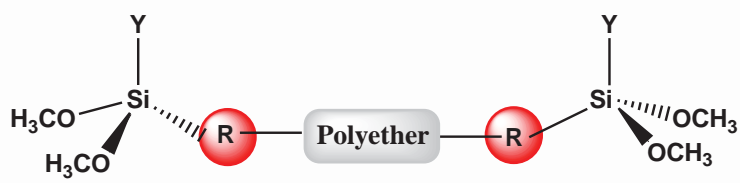

SMP1 = dimethoxysilane based polyether with $\mathrm{R}=\mathrm{CH}_{2} \mathrm{NHCOO}$

SMP2 = trimethoxysilane based polyether with $\mathrm{R}=\mathrm{C}_{3} \mathrm{H}_{6}-\mathrm{O}$ 
$\mathrm{Y}=\mathrm{CH}_{3}(\mathrm{SMP} 1)$ or $\mathrm{OCH}_{3}(\mathrm{SMP} 2)$

Fig. 2: Scheme of silyl terminated polyethers

\subsection{Chemical products}

All chemicals were purchased from Sigma Aldrich and were used as received without further purification.

Acid catalysts: Trifluoromethanesulfonic acid (HOTf, >99\%wt), Hydrochloric acid (HCl, 37\%wt), Methanesulfonic acid (MSA, >99,5\%wt), p-Toluenesulfonic acid monohydrate (PTSA, 98\%wt), Dodecylbenzenesulfonic acid (DDBSA, 95\%wt), Oxalic acid (98\%wt), Maleic acid (>99\%wt), Malonic acid (99\%wt), Chloroacetic acid (99\%wt), Glycolic acid (99\%wt), Benzoic Acid (>99,5\%wt), Acetic acid (>99,7\%wt), Propionic acid (>99,5\%wt), Lauric acid (>98\%wt), Stearic acid (95\%wt),

Amine catalysts: 1,8-Diazabicyclo[5.4.0]undec-7-ene (DBU, 98\%wt), 1,5-Diazabicyclo[4.3.0]non-5ene (DBN, 98\%wt), 1,5,7-Triazabicyclo[4.4.0]dec-5-ene (TBD, 98\%wt), 4-(Dimethylamino)pyridine (DMAP, >99\%wt), 1,4-Diazabicyclo[2.2.2]octane (DABCO, >99\%wt), Tributylamine (TBA, $>98,5 \% w t)$, Triethylamine (TEA, $\geq 99 \% w t$ ), Pyrazole (98\%wt), 1-Methylimidazole (99\%wt), Imidazole ( $>99 \% w t)$, Pyridine ( $>99 \% w t), 1,2,4$-Triazole (98\%wt) and Triethanolamine ( $>99 \% w t)$ and Metal-based catalysts: Dibutyltin dilaurate (DBTDL, 95\%wt), Zirconium(IV) propoxide (Zr(IV) $)^{\mathrm{n}} \mathrm{OPr}$, 70\%wt), Zirconium(IV) isopropoxide (Zr(IV) ${ }^{\mathrm{i}} \mathrm{OPr}$, 99,9\%wt), Zirconium(IV) butoxide (Zr(IV) $\left.)^{\mathrm{n}} \mathrm{OBu}, 80 \% w t\right)$, Titanium(IV) propoxide (Ti(IV) $\left.{ }^{\mathrm{n}} \mathrm{OPr}, 98 \% \mathrm{wt}\right)$, Titanium(IV) isopropoxide (Ti(IV) $)^{\mathrm{i}} \mathrm{OPr}$, 97\%wt), Titanium(IV) butoxide (Ti(IV) ${ }^{\mathrm{n}} \mathrm{OBu}, 97 \% \mathrm{wt}$ ), Zirconium(IV) acetylacetonate (Zr(IV)acac, 98\%wt), Aluminum acetylacetonate (Al(III)acac, 99\%wt), Iron(III) acetylacetonate (Fe(III)acac, 97\%wt), Copper(II) acetylacetonate (Cu(II)acac, 97\%wt), Calcium acetylacetonate (Ca(II)acac, 99,95\%wt), Manganese(II) acetylacetonate (Mn(II)acac).

In order to simplify tables and figures, the different catalysts are not reported in full name. When available, only corresponding abbreviations are used.

\subsection{Development of high-throughput experiment method with DBU}

SMP1 (55g) was introduced into a large plastic vessel $(185 * 95 * 15 \mathrm{~mm})$. As indicated in Fig. 3, a plate containing 96 holes was slowly disposed on the plastic tank to allow the viscous liquid to fill the 96 cavities by capillarity. By this way, 96 identical samples of prepolymer are generated without any transfer by multichannel pipette or individual weighing. Each reactor has an $8 \mathrm{~mm}$ diameter and 8mm height. Various rates of DBU 0.05\%wt (rows $\mathrm{G}$ and $\mathrm{H}$ ), 0.1\%wt (rows $\mathrm{E}$ and F), 0.3\%wt (rows $\mathrm{C}$ and D) and $0.5 \%$ wt (rows A and B) $(0.032 \mathrm{mmol}$ to $0.32 \mathrm{mmol})$ were added at the surface of each well with a multichannel pipette. The reaction begins when the mixture is in contact with moisture to allow the development of cross-linking reactions.

\subsection{General procedure for parallel screening of catalysts (HTS)}

Typically, 1mL tubes (from VWR) are placed in a plate A, containing 96 holes. In a glove box, catalysts $\left(2,25.10^{-5} \mathrm{~mol}\right)$ and THF $(50 \mu \mathrm{L})$ were added in each tubes. After closing tubes, mixtures were stirred during $1 \mathrm{~h}$ at room temperature. SMP1 (55g) was introduced in a large plastic vessel $(185 * 95 * 15 \mathrm{~mm})$. Another plate B, containing 96 holes, was slowly disposed on the plastic tank to allow the viscous liquid to fill to 96 cavities by capillarity. By this way, 96 identical samples of prepolymer are generated without any transfer by multichannel pipette or individual weighting. Each reactor has an $8 \mathrm{~mm}$ diameter and $8 \mathrm{~mm}$ height. Catalytic systems were then transferred from the plate A into the 96 micro-reactors of plate B with a multichannel pipette to form a film at the surface of 
each reactor. The reaction was performed without stirring during 1 hour (or during time t if specified) at room temperature (classical conditions in the laboratory were $55 \% \mathrm{H}_{2} \mathrm{O}$ and $23^{\circ} \mathrm{C}$ ).

\subsection{General procedure for polymerization of SMP1 in bulk}

Catalysts $\left(4,5.10^{-4} \mathrm{~mol}\right)$ and THF $(100 \mu \mathrm{L})$ were added in $1 \mathrm{~mL}$ tubes (from VWR). Mixtures were stirred during $1 \mathrm{~h}$ at room temperature. SMP1 $(10 \mathrm{~g})$ was introduced into a plastic cup (diameter 50mm and height $30 \mathrm{~mm}$ ). Catalytic systems were introduced into the plastic cup and stirred with prepolymer for 1 minute. Surface texture was evaluated by touching the surface every 5 min over a period of one hour, followed by 1 hour for 8hrs and every 24h for 1 week.

\subsection{General procedure for polymerization of SMP2 in bulk}

Catalysts $\left(9,5.10^{-5} \mathrm{~mol}\right)$ and THF $(100 \mu \mathrm{L})$ were added in $1 \mathrm{~mL}$ tubes (from VWR). Mixtures were stirred over a period of $1 \mathrm{~h}$ at room temperature. SMP2 prepolymer $(10 \mathrm{~g})$ was introduced into a plastic cup (diameter $50 \mathrm{~mm}$ and height $30 \mathrm{~mm}$ ). Catalytic systems were introduced into the plastic cup and stirred with prepolymer for 1 minute. Surface texture was evaluated by touching the surface every 5 min over a period of one hour, every 1 hour for 8hs and every 24h for 1 week.

\section{RESULTS AND DISCUSSION}

\subsection{Development and validation of high-throughput screening method}

The key point for any HTS approach is to be sure to compare all experiments, accurately performed in the same conditions: a) the amount of material must be identical in each micro-reactor, b) the ratio of catalyst/prepolymer should be the same, c) when different components are mixed together the homogeneity should be guaranteed, d) one should pay attention to avoid cross contamination between micro-reactors. We have developed a new experimental protocol specific for extremely viscous systems. The prepolymer was introduced into a large plastic vessel $(185 * 95 * 15 \mathrm{~mm})$. A plate containing 96 holes was slowly disposed on the plastic tank to allow the viscous liquid to fill the 96 cavities by capillarity. By this way, 96 identical samples of prepolymer are generated without any transfer by multichannel pipette or individual weighing. Catalytic systems were then transferred from a 96 wells plate A to the 96 micro-reactors of plate B with a multichannel pipette. The small size of the individual reactors do not allow an efficient stirring, thus there is a strong risk of having an inhomogeneous reaction mixture. Therefore, we proposed to create a film of catalysts solution at the surface of each well, instead mixing of components. All experiments were able to allow the crosslinking reactions to occur at the surface in contact with moisture under the same conditions for all experiments. Each reactor has an $8 \mathrm{~mm}$ diameter and $8 \mathrm{~mm}$ height. Thus, the height prevents any risk of catalyst migration from the surface to the bottom of each well. The screening was performed by touching the surface of each well with a multichannel tip system to visualize the physical state (viscous or gum solid state) every 5 minutes during 1 hour at room temperature. For a given time and only if the catalyst is active, the effective curing of polymers will affect both the surface and the top part of the micro-reactor as the cure depth is related to the square root of time. Thus when the surface is viscous, this means that the catalyst is inefficient. But when a gum aspect occurs (indicating surface and deep curing), this means that the catalyst is efficient. We have validated this high-throughput screening by the classification of catalysts in 3 categories (i.e.; fast, medium and slow activity). Three different rates of a well-known catalyst DBU were applied to simulate three different activity levels of catalysts (Fig. 3). 


\section{ACCEPTED MANUSCRIPT}

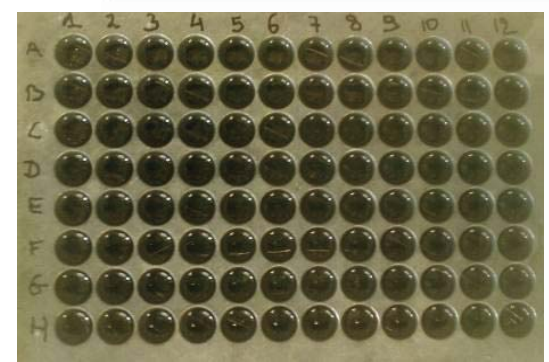

Screening with multichannel tips system by parallel approach
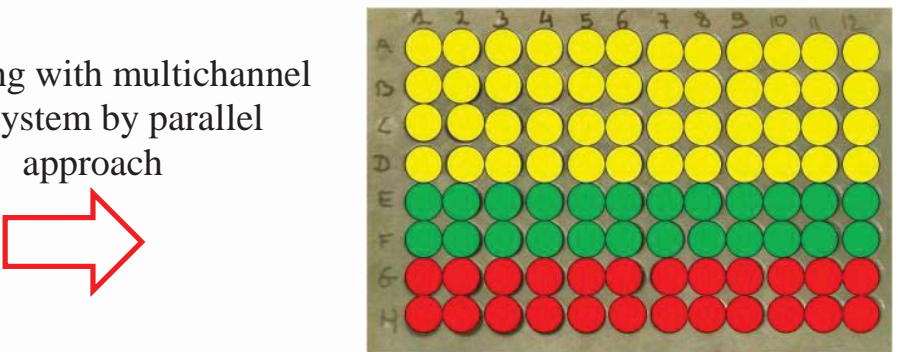

0,5\% and $0,3 \%$ wt DBU $=$ Fast (efficient between 0 and 15 minutes)

$0,1 \%$ wt DBU $=$ Medium (efficient between 15 and 30 minutes)

- $0,05 \%$ wt DBU $=$ Slow (inefficient after 1 hour)

Fig. 3: Scheme of the results obtained during the development of high-throughput screening method

This method allows the fast detection of catalytic systems with different activities. Indeed, we can see the difference with different rates of DBU, 0.5\%wt DBU is faster than $0.05 \%$ wt DBU which is slower. Moreover, the good reproducibility of the HTS method was validated because for each DBU rate, similar results were obtained. After this validation step, the HTS method was applied on several libraries to compare and to detect efficient catalysts on silyl modified polymers.

\subsection{Screening of amines library}

Amines and especially tertiary amines are well-known to catalyze polyurethane synthesis, such as DBU and TBD. These catalysts and other amines are also described by Wakabayashi et al. in their patent on alkoxysilane terminated polymers [23]. The activation mechanism is similar in these two cases. In the first case, Schwetlick et al. [24] showed that tertiary amine allows the activation of alcohol through a base catalysis mechanism to facilitate the addition of this alcohol onto an isocyanate. In the second case, Riegel et al. [25] suggested that an amine catalyst increases the dissociation of water to produce nucleophilic hydroxylanions which can attack alkoxysilane groups on a 3glycidoxylpropyl-trimethoxysilane (GPTS) molecule to accelerate hydrolysis. We can expect that the hydrolysis and condensation mechanisms to crosslink alkoxysilane terminated polymers are the same as base catalysis. To our knowledge, a comparison of the different catalytic systems under the same conditions has not been reported on alkoxysilane terminated polyethers. That is why the efficiency of several amine catalysts was determined by HTS method under the same conditions (temperature and humidity). DBTDL catalyst was used as a reference to compare its activity with amine catalysts (Fig.4). 


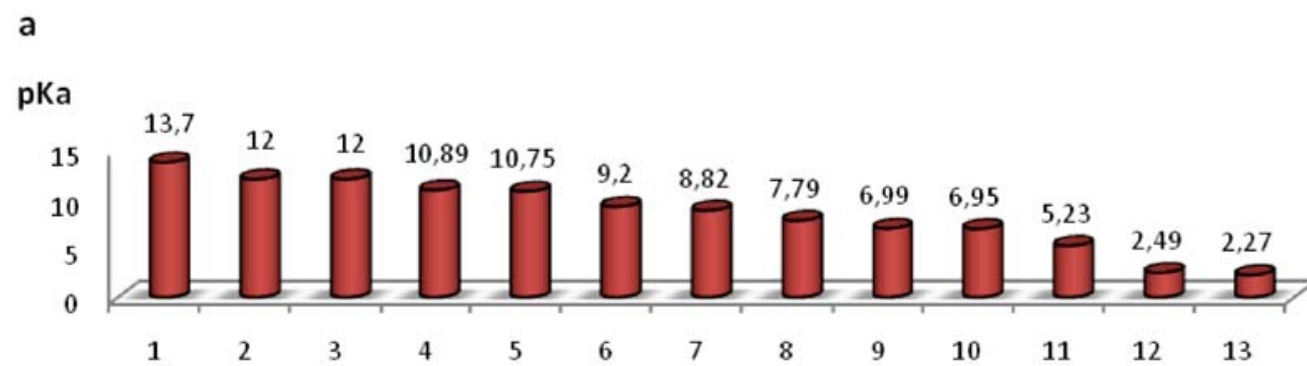

b

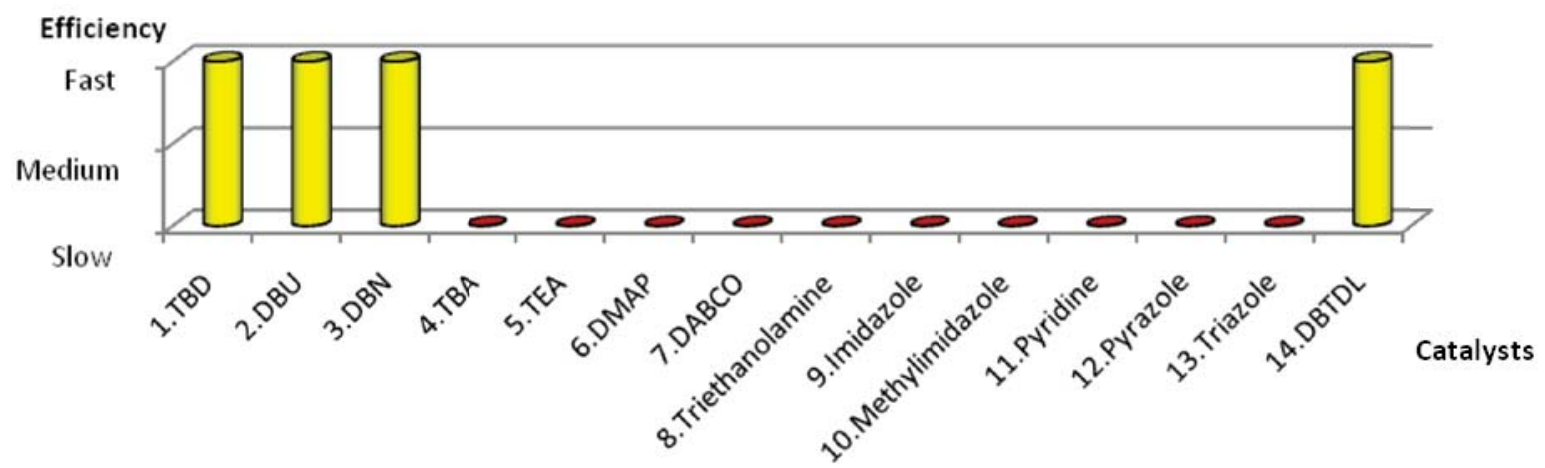

Fig. 4: a) pKa values of amine compounds and b) their efficiency on SMP1 obtained by HTS method at room temperature

The HTS method allows a fast comparison of different amines under the same conditions. Lower activities, by comparison with the reference tin catalyst DBTDL, were observed for amines with low basicity (pKa 2 to 9 in water) but also for stronger bases such as $\mathrm{NEt}_{3}$ or $\mathrm{NBu}_{3}$ (pKa around 10-11 in water). These pKa values in $\mathrm{H}_{2} \mathrm{O}$ have been presented by Perrin [26]. In fact only super bases such as DBU, DBN and TBD were really active. The very high basicity of DBN, DBU (pKa at 12) [27] and TBD (pKa at 13.7) [28], induced by the amidinium (DBU, DBN) and guanidinium (TBD) stabilization, seems to be a key point for high catalytic activity. The same differentiation, when considering pKa in acetonitrile, is observed with low activity for bases with pKa lower than 19 (TEA : 18.5 ; DABCO : 18.3) [29] and high activity for bases with pKa higher than 23 (DBU : 23.9 ; DBN : 23.4 ; TBD : 26) [30]. Cramail et al. [31] proposed a mechanism which is based on hydrogen bonding between a guanidine like TBD and an alcohol to provide nucleophilic activation. Similarly, in addition to the basicity, the potential participation of the amidinium and guanidinium moiety in the catalytic activation of the alkoxysilane hydrolysis and condensation could be proposed (Fig. 5). 


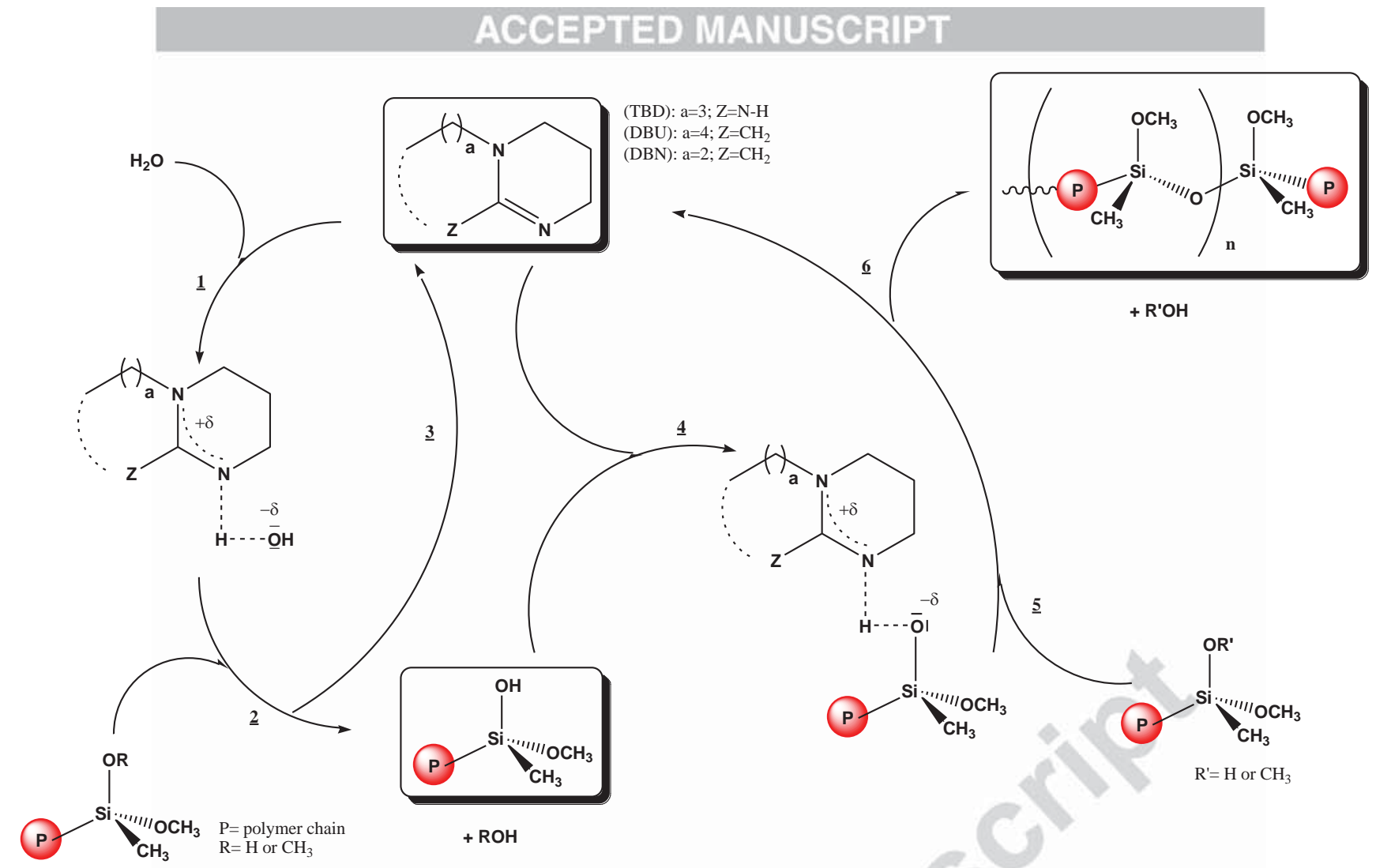

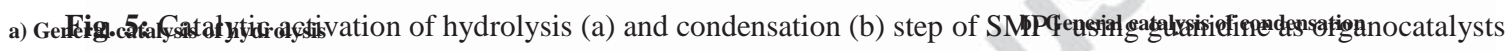

A guanidine or amidine system such as TBD, DBU or DBN can activate a water molecule (1). The nucleophilic character of hydroxyl was enriched to favor the nucleophilic substitution with an alkoxysilane group to provide a hydroxyl alkoxysilane group (2) and the regeneration of catalyst (). . Then, the catalyst system can also increase the reactivity of the hydroxyl alkoxysilane by hydrogen bonding (4). The condensation between this system and another hydroxy or alkoxysilane (ㄷ) leads to the formation of polymer or a 3D network structure (ㅁ).

\subsection{Screening of acids library}

Acids are also able to catalyze the hydrolysis and condensation of terminal alkoxysilyl groups. Brinker et al. [32] showed that in acid catalysis ( $\mathrm{HCl}$ ) on tetraethylorthosilicate (TEOS), the hydrolysis is favored compared to condensation by $\mathrm{H}^{+}$electrophilic attack on alkoxide oxygen. In this case, the silicate gel is not highly crosslinked as compared to base catalysis. Pope et al. [33] reported the effect of several inorganic acids such as $\mathrm{HF}, \mathrm{HCl}, \mathrm{HNO}_{3}, \mathrm{H}_{2} \mathrm{SO}_{4}$ on TEOS gel time, considering both hydrolysis and condensation steps. Organic acids catalysts are also very interesting with the opportunity to modulate the Bronsted or Lewis acid properties by playing with the acid functional part (carboxylic, sulfonic groups, ..) or with the other part of the molecule (aliphatic or aromatic acids, electroattractive or electrodonating substituents, ..). For instance, Ni et al. [34] showed that $\rho$-toluene sulfonic acid (PTSA) can be used to catalyze the moisture curing of an organic/inorganic mixture composed of an alkoxysilane terminated isocyanurate, 1,6-hexamethylene diisocyanate (HDI) and TEOS. Thus, an HTS method could be certainly useful to investigate a large variety of organic acid catalysts and to compare them with DBTDL as reference (Fig. 6). 


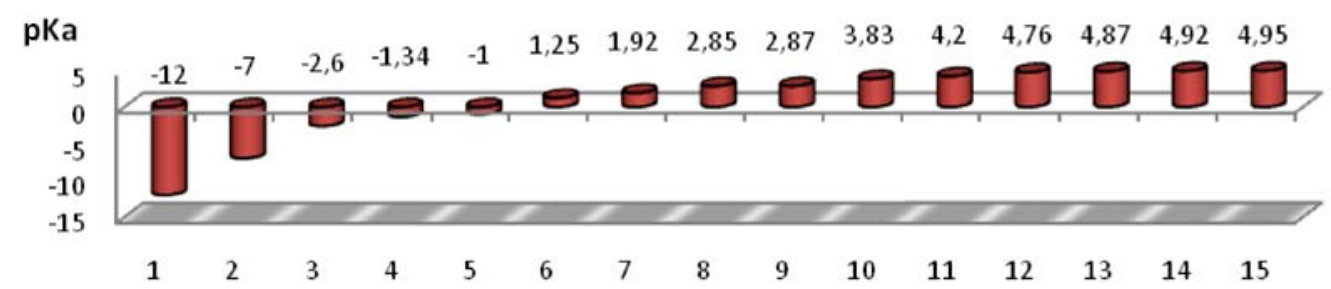

b

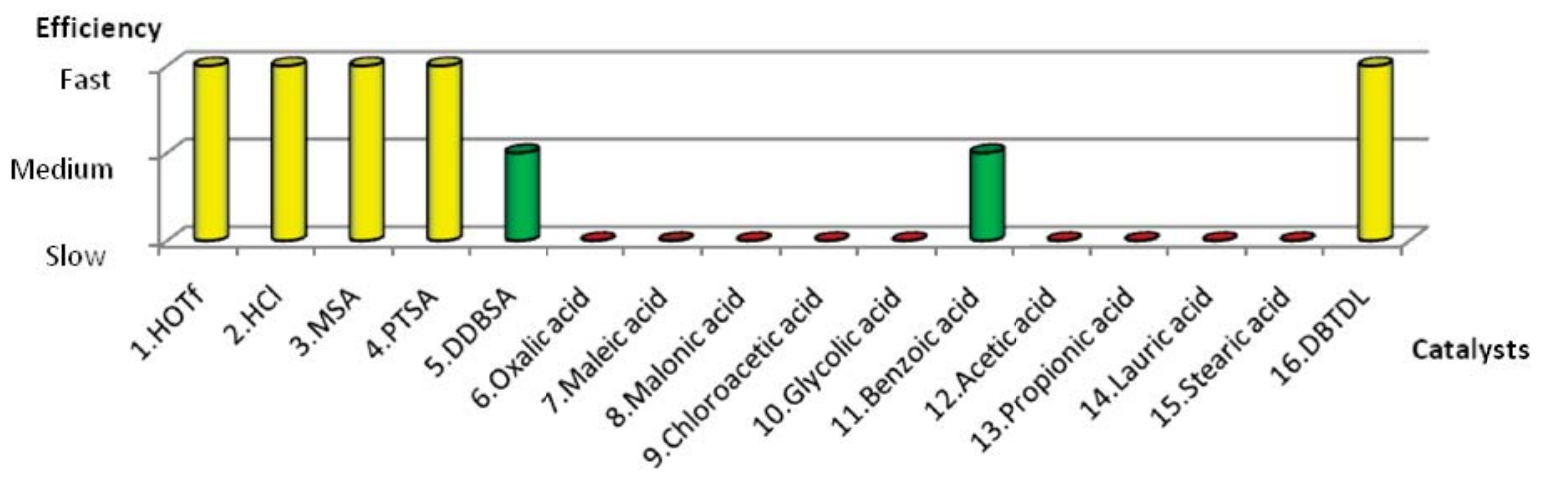

Fig. 6: a) pKa values of acid compounds and b) their efficiency on SMP1 obtained by HTS method at room temperature

A variety of organic acids were tested, including strong and weak acids, monocarboxylic acids with short and long aliphatic chains or aromatic groups, di-carboxylic acids with different methylene units as well as acids with hydroxyl or chlorine substituents. The different weak acids with relative pKa higher than 1, were medium or not efficient for the cross-linking of SMP1 by comparison with the tin reference catalyst tested under the same conditions. At room temperature and after 1 hour of reaction, all the systems involving weak acids stayed more or less viscous but did not generate the same solid gum aspect like the reference DBDTL. As expected, strong acids with low pKa [35] value such as HOTf (-12), HCl (-7), MSA (-2.6) and PTSA (-1.34) are very efficient crosslinking silyl modified polymers in less than $15 \mathrm{~min}$. These results are coherent with the fact that generally speaking and according to our HTS results, acids with a pKa value equal or less than -1 are efficient systems.

Interestingly, the HTS method led us to detect catalysts with intermediate activity able to generate the solid gum aspect but only after 15 to $30 \mathrm{~min}$. For instance, the HTS method showed that a substitution of the methyl group of PTSA (pKa -1.34) [36] by a longer dodecyl group for the DDBSA (pKa -1) [37] has a great impact on the activity. This effect could be tentatively attributed to higher hydrophobicity induced by the longer dodecyl group by comparison with a methyl group. However, the HTS method also gave an unexpected result as benzoic acid was also detected as a catalyst with medium activity like DDBSA. Only the acidic property seems not enough to explain this result because benzoic acid has a high pKa value (4.2). In comparison with other carboxylic acids like oxalic or maleic acids, it seems that the electronic delocalization through the aromatic ring for the benzoate anionic is a key factor for the catalyst efficiency. Based on the generally accepted hypothesis, indicating that the cross-linking of alkoxysiloxane is favored when using acids with low pKa value, the benzoic acid would not be evaluated. This result indicated that the HTS approach could be really interesting to detect new acidic catalyst-families.

\subsection{Screening of $\mathrm{M}-(\mathrm{OR})$ and $\mathrm{M}(\mathrm{acac})$ libraries}

In some cases, organic catalysts could present some side effects due to the residual catalyst, such as odor diffusion from the material, color modification versus time as well as migration of the catalyst from the 3D cross-linked polymer to another matrix in contact with the polymer. In addition, the organic catalysts could also affect the structure and the morphology of the final reticulated material. For instance, acid catalysts accelerate the hydrolysis step and generate more linear units than base 


\section{ACCEPTED MANUSCRIPT}

catalysts. Basic catalysts generate more dense structures and sometimes colloidal containing structures. Thus, the use of metal-containing catalysts, more particularly metal alkoxides, is also of great interest to generate polymers. Blank et al. [38] reported several organometallic compounds such as $\mathrm{Zr}(\mathrm{IV}) \mathrm{acac}, \mathrm{Zr}(\mathrm{IV}) \mathrm{O}^{\mathrm{n}} \mathrm{Bu}$ and $\mathrm{Ti}(\mathrm{IV}) \mathrm{O}^{\mathrm{n}} \mathrm{Bu}$ for the reaction between an isocyanate and a hydroxyl group. Wen et al. [39] showed that metallic alkoxides were used as precursors to form an organic/inorganic hybrid network material. In fact, the use of metal alkoxide compounds as efficient catalysts for silyl modified polymers has already been described [40]. Acetylacetonate has often been used as a chelating ligand to decrease hydrolysis and condensation reactions. Several catalysts, such as $\mathrm{Al}(\mathrm{III}) \mathrm{acac}$, have also been reported to increase the hydrolysis on methyltrimethoxysilane and tetramethoxysilane [41], as well as Fe(III)acac on silyl terminated polymers [42]. However, these reports do not provide a clear comparison between each catalyst for the same substrate, thus it is practically impossible to extract relative reactivity data. In order to compare the efficiency of several metal-containing catalysts we have applied the HTS method and compared to DBTDL as reference under the same conditions (Fig. 7).

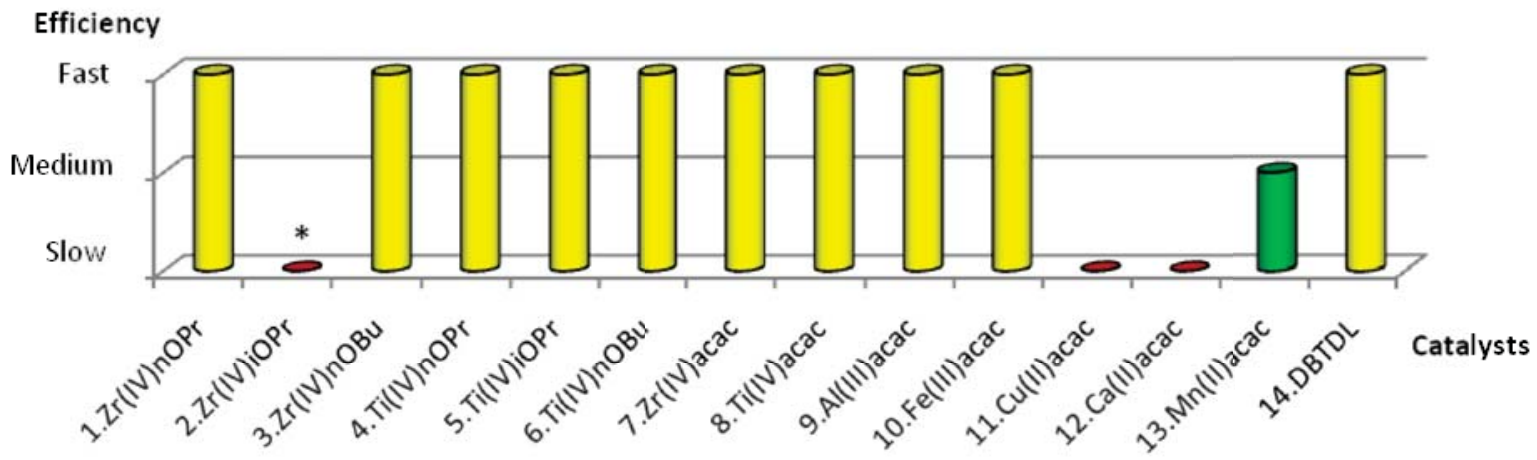

Fig. 7 Efficiency of metallic compounds on SMP1 obtained by HTS method at room temperature *= heterogeneous mixture

All metallic alkoxides tested here are very efficient on silyl terminated polyethers except for $\mathrm{Zr}(\mathrm{IV}) \mathrm{O}^{\mathrm{i}} \mathrm{Pr}$ which is inefficient. It seems that the $\mathrm{Zr}(\mathrm{IV}) \mathrm{O}^{\mathrm{i}} \mathrm{Pr}$ is so reactive with moisture that it is too quickly deactivated and has no time to react with Si-OMe bonds inducing an heterogeneous system. In the literature, Pandey et al. [43] used a combination of $\mathrm{Zr}(\mathrm{IV}) \mathrm{OiPr}$ and dichloroacetic acid to decrease the reaction of zirconium tetraisopropoxide with moisture. $\mathrm{Ti}(\mathrm{IV}) \mathrm{O}^{\mathrm{i}} \mathrm{Pr}$ is effective as a catalyst but this result could be explained by the lower reactivity of Ti(IV)OR than $\mathrm{Zr}(\mathrm{IV}) \mathrm{OR}$ reported by Wen et al. [44]. As expected, the results show that acetylacetonate systems with $\mathrm{Zr}$, $\mathrm{Ti}, \mathrm{Al}$ and $\mathrm{Fe}$ are very efficient. Copper and Calcium acetylacetonate systems are inefficient after 1 hour. Interestingly, a manganese system has medium efficiency. This result was very difficult to predict, thus showing the potential benefits of a fast parallel comparison method.

In conclusion, the development of a semi-quantitative high-throughput screening method allows the classification of different catalytic systems according to their efficiency. All catalysts were compared under rigorous identical conditions: geometry and size of micro-reactors, moisture exposure and time for parallel testing. Thus, only differences in catalytic properties will affect the surface or depth curing [45] of polymer. This method is applicable to different types of catalysts like organic bases or acids as 


\section{ACCEPTED MANUSCRIPT}

well as for metal-containing catalysts. This can be applied to detect new catalysts by testing 96 catalysts under the same conditions. It is also to spend time on more focused studies, but only on the best candidates for target applications.

\subsection{Skinning time of some efficient catalysts in bulk}

The HTS method allowed the ranking of different catalytic systems into 3 categories (fast, medium and slow catalysts) according to their relative activity versus the reference catalyst DBDTL. The second step is to measure their 3D solid cross-linking time values compared to DBTDL (Table 1) in order to conclude if DBTDL can be substituted.

Table 1. 3D crosslinked gum solid state time of efficient catalysts in bulk by classical method at room temperature on SMP1 prepolymer

\begin{tabular}{cc}
\hline Catalysts & Gum solid state time (min) \\
\hline DBTDL & 150 \\
\hline HOTf & instantaneous \\
HCl & instantaneous \\
MSA & instantaneous \\
PTSA & instantaneous \\
DDBSA & instantaneous \\
Benzoic acid & 4320 \\
\hline DBU & 10 \\
TBD & instantaneous \\
\hline Zr(IV) $\mathrm{n}$ OPr & 30 \\
Ti(IV) ${ }^{\mathrm{n} O P r}$ & 30 \\
\hline Zr(IV)acac & 120 \\
Ti(IV)acac & 60 \\
Al(III)acac & 390 \\
Fe(III)acac & 105 \\
Mn(II)acac & 75 \\
\hline
\end{tabular}

Using the same conditions, temperature, humidity and rate of catalytic system, the reactions were performed in bulk condition for $10 \mathrm{~g}$ of prepolymer SMP1 and a fast mixing of the mixture. Concerning the acids catalysts HOTf, HCl, MSA, PTSA and DDBSA are much more active than DBDTL as they induced an instantaneous 3D cross-linking. However, these systems are not very useful in bulk conditions or for adhesive applications as a setting time is necessary to generate a homogeneous mixture for bulk reactions and/or to apply the adhesive materials onto a surface. If the final application does not need a very short setting time, the benzoic acid could be applied according to a lower toxicity than the tin catalyst DBDTL. In our application, a setting time of few minutes is necessary to apply adhesive materials on a surface. Benzoic acid could be interesting due to the high toxicity of DBTDL. For bases systems, if TBD generated an instantaneous cross-linking very difficult to control, the DBU catalyst presented very interesting properties with a shorter time for reticulation than DBDTL and a lower toxicity. Metal alkoxides also present high potential as they are faster than the tin catalysts with a lower toxicity. For longer setting time it could also be interesting to use the less reactive metal acac complexes as they are less moisture sensitive than metal alkoxides. 


\section{ACCEPTED MANUSCRIPT}

\begin{tabular}{cc}
\hline DBTDL & 48 \\
\hline HOTf & instantaneous \\
HCl & 0.1 \\
MSA & 0,15 \\
PTSA & instantaneous \\
DDBSA & instantaneous \\
Benzoic acid & no \\
\hline DBU & 3 \\
TBD & $8<\mathrm{t}<24^{*}$ \\
\hline Zr(IV) ${ }^{\mathrm{n} O P r}$ & $8<\mathrm{t}<24^{*}$ \\
Ti(IV) ${ }^{\mathrm{n} O P r}$ & 72 \\
\hline Zr(IV)acac & $8<\mathrm{t}<24^{*}$ \\
Ti(IV)acac & $8<\mathrm{t}<24^{*}$ \\
Al(III)acac & $8<\mathrm{t}<24^{*}$ \\
Fe(III)acac & no \\
Mn(II)acac & \\
\hline
\end{tabular}

\footnotetext{
*: Solid gum state reaching time was evaluated at room temperature every 5 min during one hour and every 1 hour until 8 h. Days after and early morning, skinning time was evaluated every $24 \mathrm{~h}$ until 1 week.
}

Table 2. Skinning time of efficient catalysts in bulk by classical method at room temperature on SMP2

The hits detected by the HTS approach were also tested for another prepolymer SMP2 (Fig. 2). In fact, the SMP2 and SMP1 are methoxysilane terminated polymers. In the case of urethanedimethoxysilane (SMP1), the electron donor (nitrogen atom with free electron pair) is attached to a silicon atom via only one methylene group as spacer. In addition, the methyl group attached to the silicon atom can play an important role by inductive effect. Indeed, the electron density is shifted to the silicon atom and the $\mathrm{Si}-\mathrm{O}\left(\mathrm{CH}_{3}\right)$ bond is weakened. Thus for SMP1, the time of cross-linking reaction decreases due to the activation of alkoxide group. That is why the alkoxide groups split off more easily and hydrolysis is accelerated for SMP1. We envisioned that if the SMP2 is less reactive than SMP1, it is not necessary to screen all of the catalysts again for the SMP2, because an inefficient catalyst on SMP1 will be inefficient too on SMP2. Times to reach the gum aspect of different hits catalysts were determined on SMP2 (Table 2). HOTf, PTSA and DDBSA were no longer usable as they induced out of control reticulation. However, $\mathrm{HCl}$ and MSA are approximately 200 to 300 times more efficient than DBTDL for the cross-linking of SMP2 and benzoic acid which is slow on SMP1 is inefficient on SMP2. Benzoic acid and DBU did not allow the cross-linking, these results are not surprising as these two catalysts presented a medium activity for the SMP1. In the same way, the TBD, much active for SMP1 is under control for the SMP2 and is 16 times more efficient than DBTDL. Metallic alkoxides and metal acetylacetonate are also more efficient than the tin catalyst, excepted for $\mathrm{Zr}(\mathrm{IV})$ acac and $\mathrm{Mn}(\mathrm{II})$ acac which are inactive.

\section{CONCLUSIONS}

A new HTS method has been developed to allow fast comparison of catalysts for very viscous prepolymers where it has been applied to the study of silyloxane prepolymer cross-linking. The method is compatible with different catalyst families, including inorganic and organic bases, acids and also for metal containing complexes. The method allows to compare and to extract different hits which were analysed in a second step to extract their relative solid state reaching time. DBU, metallic alkoxides and metal acetylacetonate systems could be used to substitute organotin catalysts on silylated activated prepolymers. For inactivated ones, only TBD, HCl, MSA, metal alkoxides and 


\section{ACCEPTED MANUSCRIPT}

several metal acetylacetonate systems have shown catalytic activity. This HTS method will be used to detect new catalysts to substitute organotin catalysts to generate ecofriendly adhesive materials.

\section{ACKNOWLEDGEMENTS}

The authors gratefully acknowledge CNRS, ANRT, BOSTIK and TOTAL for their financial support.

\section{REFERENCES AND NOTES}

[1] S. Maudgal, T.L. St Clair, Int. J. Adhesion and Adhesives 4 (1984) 129-132.

[2] H.H. Huang, G.L. Wilkes, Polymer 30 (1989) 2001-2012.

[3] P. Liu, J. Song, L. He, X. Liang, H. Ding, Q. Li, European Polymer Journal 44 (2008) 940-951.

[4] A. Serier, J.P. Pascault, T.M. Lam, J. Polym. Sci. Chem. 29 (1991) 1125-1131.

[5] D. Saikia, C.G. Wu, J. Fang, L.D. Tsai, H.M. Kao, J. of Power Sources 269 (2014) 651-660.

[6] H. Schmidt, B. Seiferling, B. Mater. Res. Soc. Symp. Proc. 73 (1986) 739-750.

[7] E. Alyamac, H. Gu, M.D. Soucek, S. Qiu, R.G. Buchheit, Prog. in Organic Coatings 74 (2012) 67-81.

[8] H. Maleki, L. Duraes, A. Portugal, J. of Non-Crystalline Solids 385 (2014) 55-74.

[9] M. Alexandre, P. Dubois, Materials Science and Engineering 28 (2000) 1-63.

[10] F. Surivet, T.M. Lam, J.P. Pascault, Q.T. Pham, Macromolecules 25 (1992) 4309-4320.

[11] H. Schmidt, H. Scholze, A. Kaiser, J. of Non-Crystalline Solids 63 (1984) 1-11.

[12] R.S. Burkhalter, C. L. Hogue, D.L. Smith, S.M. Sonner, M.J. Winningham, R.E. Yougman, J. of Organometallic Chemistry 724 (2013) 213-224.

[13] H. Kaddami, F. Surivet, J.F. Gérard, T.M. Lam, J.P Pascault, J. of Inorganic and Organometallic Polymers 4 (1994) 183-198.

[14] C.J. Brinker, G.W. Scherer, J. of Non-Crystalline Solids 70 (1985) 301-322.

[15] I. Matsuyama, S. Satoh, M. Katsumoto, K. Susa, J. of Non-Crystalline Solids 135 (1991) 22-28.

[16] Patent of BOSTIK 2012/0165453 A1 (2012).

[17] J.C. Meredith, J. J. Mater. Chem. 18 (2009) 35-45.

[18] B. Jandeleit, D.J. Schaefer, T.S. Powers, H.W. Turner, W.H. Weinberg, Angew. Chem. Int. Ed. 38 (1999) 2494-2532.

[19] O. Lavastre, I. Illitchev, G. Jegou, P.H. Dixneuf, J. Am. Chem. Soc. 124 (2002) 5278-5279.

[20] M. Reetz, Angew. Chem. Int. Ed. 40 (2001) 284-310.

[21] S. Garbacia, R. Touzani, O. Lavastre, J. Comb. Chem. 6 (2004) 297-300.

[22] A.M. Porte, J. Reibenspies, K. Burgess, J. Am. Chem. Soc. 120 (1998) 9180-9187.

[23] US 8,124,690 B2 (2012).

[24] K. Schwetlick, R. Noack, F. Stebner, J. Chem. Soc. Perkin Trans. 2 (1994) 599-608.

[25] B. Riegel, S. Blittersdorf, W. Kiefer, S. Hofacker, M. Müller, G. Schottner, J. of Non-Crystalline Solids 226 (1998) 76-84.

[26] D.D. Perrin, Dissociation Constants of Organic Bases in Aqueous Solution, Butterworths, London, 1965; Supplement, 1972.

[27] D. Granitza M. Beyermann, H. Wenschuh, H. Haber, L.A. Carpino, G.A. Truran, M. Bienert., J. Chem. Soc. Chem. Commun. (1995) 2223-2224.

[28] V. Verdolino, R. Cammi, B.H. Munk, H.B. Schlegel, J. Phys. Chem. B 112 (2008) 16860-16873.

[29] I. Kaljurand, A. Kütt, L. Sooväli, T. Rodima, V. Mäemets, I. Leito, I.A. Koppel, J. Org. Chem. 70 (2005) 1019-1028.

[30] J.E. Taylor, S.D. Bull, J.M.J. Williams, Chem. Soc. Rev. 41 (2012) 2109-2121.

[31] J. Alsarraf, Y.A. Ammar, F. Robert, E. Cloutet, H. Cramail, Y. Landais, Macromolecules 45 (2012) 22492256. 


\section{ACCEPTED MANUSCRIPT}

[32] C.J. Brinker, K.D. Keefer, D.W Schaefer, C.S. Ashley, J. of Non-Crystalline Solids 48 (1982) 47-64.

[33] E.J. Pope, J.D. Mackenzie, J. of Non-Crystalline Solids 87 (1986) 185-198.

[34] H. Ni, A.D. Skaja, M.D. Soucek, Prog. In Organic Coatings 40 (2000) 175-184.

[35] E. Raamat, K. Kaupmees, G. Ovsjannikov, A. Trummal, A. Kütt, J. Saame, I. Koppel, I. Kaljurand, L. Lipping, T. Rodima, V. Pihl, I.A. Koppel, I. Leito, J. Phys. Org. Chem. 26 (2013) 162-170.

[36] M.D. Clark, S. Subramanian, R. Krishnamoorti, J. of Colloid and Interface Science 354 (2011) 144-151.

[37] T. Namani, P. Walde, Langmuir 21 (2005) 6210-6219.

[38] W.J. Blank, Z.A. He, E.T. Hessell, Prog. in Organic Coatings 35 (1999) 19-29.

[39] S. Diré, F. Babonneau, J. of Non-Crystalline Solids 167 (1994) 29-36.

[40] EP 1,746,134 A1 (2007).

[41] Z. Zhang, S. Sakka, J. of Sol-Gel Science and Technology 16 (1999) 209-220.

[42] WO 2013/071078 A1 (2013).

[43] A. Pandey, A. Pandey, W.J. Parak, P. Mayer, Inorganica Chimica Acta 359 (2006) 4511-4518.

[44] J. Wen, G.L. Wilkes, Chem. Mater. 8 (1996) 1667-1681.

[45] J. Comyn, J. Day, S.J. Shaw, J. Adhesion 66 (1998) 289-301. 